\title{
Improvement of Adhesive Strength of Poly(tetrafluoroethylene) Plates through Oxygen Plasma Treatment and Subsequent Photografting of Methacrylic Acid
}

\author{
Kazunori Yamada*, Marie Tachi, Yuji Kimura \\ Department of Applied Molecular Chemistry, College of Industrial Technology, Nihon University, Narashino, Japan
}

Email address:

yamada.kazunori@nihon-u.ac.jp (K. Yamada)

${ }^{*}$ Corresponding author

\section{To cite this article:}

Kazunori Yamada, Marie Tachi, Yuji Kimura. Improvement of Adhesive Strength of Poly(tetrafluoroethylene) Plates through Oxygen Plasma Treatment and Subsequent Photografting of Methacrylic Acid. International Journal of Materials Science and Applications.

Vol. 7, No. 1, 2018, pp. 18-27. doi: 10.11648/j.ijmsa.20180701.14

Received: September 12, 2017; Accepted: September 26, 2017; Published: January 5, 2018

\begin{abstract}
Methacrylic acid (MAA) was grafted onto the surface of a poly(tetrafluoroethylene) (PTFE) plate by the combined use of the plasma treatment and photografting, and the adhesive strength between the MAA-grafted PTFE (PTFE-g-PMAA) plates with the same grafted amounts was investigated in relation to the location of grafting as well as the wettability and water absorptivity. The grafted amount at which the substrate breaking occurred at lower grafted amounts for the PTFE-g-PMAA plates prepared by the plasma treatment for shorter times before the photografting and by the photografting at higher monomer concentrations and/or at lower monomer concentrations after the plasma treatment. These grafting conditions are found to be factors affecting the location of photografting, the thickness and water absorptivity of the grafted layer, and wettability of the surface of the grafted layer. The substrate breaking was observed at the minimum grafted amount (about $2.2 \mu \mathrm{mol} / \mathrm{cm}^{2}$ ) for the PTFE-g-PMAA plates prepared at $2.0 \mathrm{M}$ and $60^{\circ} \mathrm{C}$ after the plasma treatment for $10 \mathrm{~s}$ and at $2.0 \mathrm{M}$ and $40^{\circ} \mathrm{C}$ after the plasma treatment for $120 \mathrm{~s}$. The obtained results support that the combination of the oxygen plasma treatment with photografting of MAA is an effective procedure to enhance the adhesivity of the PTFE surface.
\end{abstract}

Keywords: Poly(tetrafluoroethylene), Plasma Treatment, Photografting, Methacrylic Acid, Surface Modification, Surface Analysis, Adhesive Strength

\section{Introduction}

Poly(tetrafluoroethylene) (PTFE) possesses good chemical and physical properties, such as thermal stability, chemical resistance, low water absorptivity, and low electrical conductivity $[1,2]$. On the other hand, the hydrophobicity frequently limits the use of PTFE in adhesion-related and/or wettability-related fields [3, 4]. Therefore, hydrophilization of the surfaces of the PTFE materials is of great importance to expand their industrial application [5].

Surface modification of PTFE materials for enhancement of wettability or adhesivity includes the chemical and physical procedures, such as basic and strongly acidic/oxidizing solutions [6], plasma treatment with non-polymerizable gases or their mixture gases [7-9], irradiation of ionizing or high-energy rays $[10,11]$, graft polymerization by irradiation of $\mathrm{Co}^{60}$ ray $[12,13]$ and abrasion [6]. Of them, the plasma treatment has been widely used to make the PTFE surfaces hydrophilic without considerably affecting bulk properties [14-17]. However, the modified surface properties may be gradually regressed by the time due to thermal movement of substrate polymer chain segments [18, 19]. Compared with this, the grafting polymerization is an effective surface modification technique against the time-dependent regression. The key advantage of the grafting polymerization is that modified properties can be conferred to the substrate polymer surfaces by a choice of functional or hydrophilic monomers and durable surface modification can be achieved. 
However, it is difficult to graft hydrophilic monomers onto the PTFE surfaces by photografting alone without any pretreatment mainly due to chemical stability, higher than polyethylene (PE) and polypropylene (PP) [20-22]. So far, the studies have been carried out on the enhancement of the adhesion and autohesion, or adhesive-free adhesion, of the low-density PE (LDPE), high-density PE (HDPE), and PP plates through the photografting of methacrylic acid (MAA), acrylic acid (AA), methcarylamide (MAAm), and 2-(dimethylamino)ethyl methacrylate (DMAEMA) [23, 24]. In addition, the grafted layers formed on these polymer substrates possess good water absorptivity, strongly depending on the location of photografting and/or length of grafted polymer chains. These characteristics can be controlled by the temperature and initial monomer concentration on the photografting $[21,22,25]$. As a result, the substrate breaking was observed for adhesion or autohesion of the grafted LDPE, HDPE, and PP plates at a high grafted amount [20-22, 25-27]. This means that the strengths of adhesion and autohesion exceed the ultimate strength of each polymer substrate. However, as far as we know, unfortunately, methacrylic or acrylic hydrophilic monomers are little grafted onto a PTFE surface by the photografting technique without any pretreatment. In fact, the radiation grafting has been used for surface modification of PTFE and poly(ethylene-alt-tetrafluoroethylene) (PETFE) [12, 28-31].

Therefore, the plasma treatment is selected as a pretreatment procedure for forming an active site for photografting. When a PTFE surface is plasma-treated with non-polymerizable gases, such as $\mathrm{O}_{2}, \mathrm{~N}_{2}$, and $\mathrm{Ar}$, followed by exposure to oxygen gas or air, various oxygen-containing functional groups are generated on the surface [32-34]. Of the oxygen-containing functional groups formed on the surfaces a hydroperoxide group is known to be thermally decomposed into an active site of grafting $[35,36]$.

In this study, after the surface activation of the PTFE plates by the oxygen plasma treatment, MAA was photografted onto the plasma-treated PTFE plates. The surface wettability of the MAA-grafted PTFE (PTFE-g-PMAA) plates and water absorptivity of the grafted layers formed on the PTFE plates were investigated as a function of the treatment conditions, such as the plasma treatment time and the monomer concentration and temperature on the photografting. Finally, the adhesive strength between the PTFE-g-PMAA plates with the same grafted amounts was investigated with an epoxy-type adhesive in relation to the location of grafting as well as the wettability and water absorptivity

\section{Experimental}

\subsection{Materials}

A PTFE plate of $0.50-\mathrm{mm}$ thickness was purchased from Nichias Corp., (Tokyo, Japan) and used as polymer substrate for photografting. MAA and benzophenone (BP) as sensitizer were purchased from Wako Pure Chemical (Tokyo, Japan) and used as received. A commercially available adhesive, a two-component type epoxy adhesive, “Araldite ${ }^{\circledR}$ " (type: AR-R30), was used for adhesion of the PTFE-g-PMAA plates. The nominal curing time of this adhesive is $3 \mathrm{~h}$ at room temperature.

\subsection{Plasma Treatment and Photografting}

The PTFE plates cut into 6.5-cm length and 2.4-cm width were washed with water, methanol, and acetone by turns, to remove contaminants from the surfaces, and then dried under reduced pressure. The plasma treatment of the PTFE plates was performed by a Shimadzu LCVD 20-type plasma apparatus (Kyoto, Japan) [37, 38]. The PTFE plates were fixed on the sample holder located between the plane electrodes. The distance between the sample holder and each plane electrode was set at $2.5 \mathrm{~cm}$. Then, pure oxygen gas was flowed into the bell jar at $20 \mathrm{~cm}^{3} / \mathrm{min}$ for a few minutes to purge the air so as to keep the inner pressure at 0.05 Torr $(6.66$ $\mathrm{Pa})$. Oxygen plasma generated at the output of $200 \mathrm{~W}$ and frequency of $15 \mathrm{kHz}$ was applied to one side of each PTFE plate for $10-180 \mathrm{~s}$, while the sample holder was rotated at 60 rpm. Then, the plasma-treated PTFE plates were placed in the oxygen atmosphere for $30 \mathrm{~min}$ at room temperature to generate oxygen-functional groups.

In acetone, $0.25 \mathrm{~g}$ of $\mathrm{BP}$ was dissolved and made up to 50 $\mathrm{cm}^{3}$ (concentration: $0.5 \mathrm{w} / \mathrm{v} \%$ ). The plasma-treated PTFE plates were immersed in this BP solution for $1 \mathrm{~min}$, and then acetone was evaporated at room temperature to coat $\mathrm{BP}$ on their surfaces [20-23]. Aqueous MAA solutions were prepared at 1.0-2.0 $\mathrm{M}$ and deaerated under reduced pressure for a few minutes. The plasma-treated PTFE plates were vertically placed into the MAA solutions at $65 \mathrm{~cm}^{3}$ in the Pyrex glass tubes. The photografting of MAA was performed by irradiating UV rays emitted from a $400 \mathrm{~W}$ high pressure mercury lamp at $60^{\circ} \mathrm{C}$ [20-23]. During the irradiation, each glass tube was revolved around the high-pressure mercury lamp while rotating. In addition, the photografting of MAA onto the PTFE plate was also performed in the temperature range from 40 to $70^{\circ} \mathrm{C}$ in a $2.0 \mathrm{M}$ monomer solution after the plasma treatment for $120 \mathrm{~s}$. Unless otherwise noted, the PTFE-g-PMAA plates were prepared by the plasma treatment for $120 \mathrm{~s}$ and subsequent photografting at $2.0 \mathrm{M}$ and $60^{\circ} \mathrm{C}$.

After the photografting, the PTFE plates were thoroughly washed with pure water to remove homopolymers attached to their surfaces and unreacted monomers, and then dried under reduced pressure. The grafted amount was calculated in $\mu \mathrm{mol} / \mathrm{cm}^{2}$ from the weight increase of the PTFE plates after the photografting and the area of the plasma-treated surfaces $\left(c a .15 .6 \mathrm{~cm}^{2}\right)$. Although the grafted amount was calculated in $\mathrm{wt} \%$ or $\mathrm{mmol} / \mathrm{g}$ in other articles [39-42], the unit of $\mu \mathrm{mol} / \mathrm{cm}^{2}$ was used in this study because of the fact that the grafted amount in $\mu \mathrm{mol} / \mathrm{cm}^{2}$ is independent of the thickness, density, or weight of the PTFE plate [20-22, 25-27].

\subsection{Modified Hydrophilic Properties}

Hydrophilic properties of the PTFE-g-PMAA plates were estimated from the water wettability and water absorptivity $[21,22]$. The contact angles for water on the surfaces of the 
PTFE-g-PMAA plates were measured by the sessile drop method at $25^{\circ} \mathrm{C}$ with a Kyowa Kagaku TYP-QI-type goniometer (Tokyo, Japan) and the $\cos \theta$ values were obtained from the average values. The PTFE-g-PMAA plates were immersed in water at $30^{\circ} \mathrm{C}$ for $24 \mathrm{~h}$. Then, the PTFE-g-PMAA plates were taken out of water, blotted with a filter paper to remove water attached to their surfaces, and weighed as quickly as possible $[38,43]$.

\subsection{XPS Analysis}

The XPS high-resolution spectra of carbon $\left(\mathrm{C}_{1 \mathrm{~s}}\right)$, oxygen $\left(\mathrm{O}_{1 \mathrm{~s}}\right)$, and fluorine $\left(\mathrm{F}_{1 \mathrm{~s}}\right)$ of an untreated PTFE plate, a PTFE plate plasma-treated for $120 \mathrm{~s}$, and a PTFE-g-PMAA plate with the grafted amount of $2.25 \mu \mathrm{mol} / \mathrm{cm}^{2}$ were recorded on a Shimadzu ESCA-3400 spectrophotometer (Kyoto, Japan) with $\mathrm{MgK} \alpha(1253.6 \mathrm{eV})$ source operating at $8 \mathrm{kV}$ and $20 \mathrm{~mA}$ $[38,44,45]$.

\subsection{Cross Section Observation}

The thickness of the grafted layers formed on the PTFE plate was determined through the cross section observation of the PTFE-g-PMAA plates with a Keyence VH-5500 optical microscopy (Osaka, Japan). The PTFE-g-PMAA plates cut into about $5 \mathrm{~mm}^{2}$ were immersed in an aqueous toluidine blue (TB) solution at $25 \mathrm{mg} / 100 \mathrm{~cm}^{3}$ for about $5 \mathrm{~min}$ to stain the grafted layers. After immersion, the samples were washed with water several times, and then dried under reduced pressure. The cross sections of the stained PTFE-g-PMAA samples were photographed through an optical microscope of
200 magnifications [22]. Then, the thicknesses of the grafted layers (stained part) and ungrafted layers (unstained part) were measured at least at five locations and the mean values were calculated. The relation of these thicknesses is expressed as Eq. (1):

the thickness of PTFE-g-PMAA plate $=$ thickness of grafted layer + thickness of ungrafted layer

Here, the thickness of the PTFE plate was $500 \mu \mathrm{m}$.

\subsection{Tensile Shear Adhesive Strength Measurements}

The PTFE-g-PMAA plates were cut into $3.0-\mathrm{cm}$ length and $1.2-\mathrm{cm}$ width. The base and the curing components of "Araldite ${ }^{\circledR}$ " were thoroughly mixed at the weight ratio of $1: 1$. Both surfaces of two pieces of the PTFE-g-PMAA plates with the same grafted amounts were coated with the adhesive component to provide a $1.2 \times 1.2 \mathrm{~cm}^{2}$ overlapping surface. The extruded adhesive components were mostly removed and the amount of mixed component was $0.74 \mathrm{mg} / \mathrm{cm}^{2}$ on average from the weight increase by application of adhesives. The adhesive component was cured by applying the load of 0.5 $\mathrm{kg} / \mathrm{cm}^{2}$ at $60^{\circ} \mathrm{C}$ for $24 \mathrm{~h}$. The force to separate the adhesive joints was measured at the strain rate of $3 \mathrm{~mm} / \mathrm{s}$ by an Orientec universal testing machine STA 1225 (A\&D company Ltd., Tokyo, Japan), and the values of tensile shear adhesive strength were calculated by dividing the force by the overlapped area.

\section{Results and Discussion}

\subsection{Plasma Treatment and Photografting}
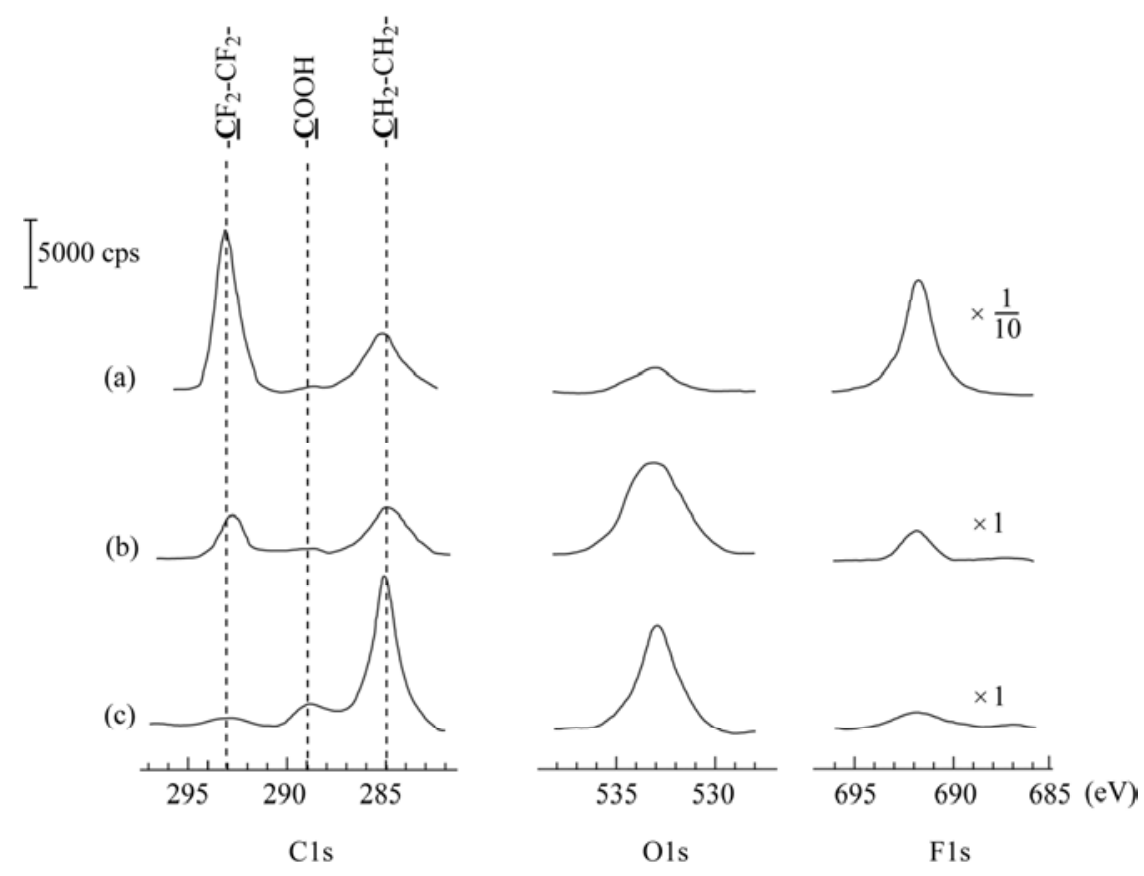

Figure 1. The C1s, O1s, and F1s core level spectra of (a) an untreated PTFE plate, (b) a PTFE plate plasma-treated for $120 \mathrm{~s}$, and (c) a PTFE-g-PMAA plate with $2.25 \mu \mathrm{mol} / \mathrm{cm}^{2}$. 
The PTFE-g-PMAA plates were prepared by combined use of plasma treatment with the photografting. Figure 1 shows the typical XPS high-resolution spectra of $\mathrm{C}_{1 \mathrm{~s}}, \mathrm{O}_{1 \mathrm{~s}}$, and $\mathrm{F}_{1 \mathrm{~s}}$ for (a) an untreated PTFE plate, (b) a PTFE plate plasma-treated for $120 \mathrm{~s}$, and (c) a PTFE-g-MAA plate with the grafted amount of $2.25 \mu \mathrm{mol} / \mathrm{cm}^{2}$. The XPS high-resolution $\mathrm{C}_{1 \mathrm{~s}}$ spectrum for the untreated PTFE plate showed a peak at 292.5 $\mathrm{eV}$ assigned to $-\underline{C F}_{2}-\mathrm{CF}_{2}-[46-48]$ and an $\mathrm{F}_{1 \mathrm{~s}}$ peak at $690 \mathrm{eV}$ with a minor peak at $285 \mathrm{eV}$ probably due to small contamination [48]. When the PTFE surface was plasmatreated for $120 \mathrm{~s}$, the intensity of the peak at $292.5 \mathrm{eV}$ and the $F_{1 s}$ peak declined and overlapped small peaks appeared in the region of 286-289 eV as shown in Figure 1 (b). This suggests that some fluorine atoms were depleted from the PTFE surface and several kinds of oxygen-containing functional groups were generated by the plasma treatment [35-37]. In addition, a peak assigned to - $\underline{\mathrm{COOH}}$ sharply appeared at $289 \mathrm{eV}$ by grafting MAA onto a plasma-treated PTFE plate [38, 39]. This indicates that MAA was successfully grafted with the PTFE surfaces. However, the $F_{1 s}$ peak at $692 \mathrm{eV}$ and the $\mathrm{C}_{1 \mathrm{~s}}$ peak at $292.5 \mathrm{eV}$ were observed for the PTFE-g-PMAA plates. This denotes that even when the PTFE-g-PMAA plates with high grafted amounts were obtained, a small amount of PTFE chains are present on the surfaces of the grafted layers. In other words, the layers of grafted PMAA chains containing a small amount of PTFE chains were formed on the PTFE surface.
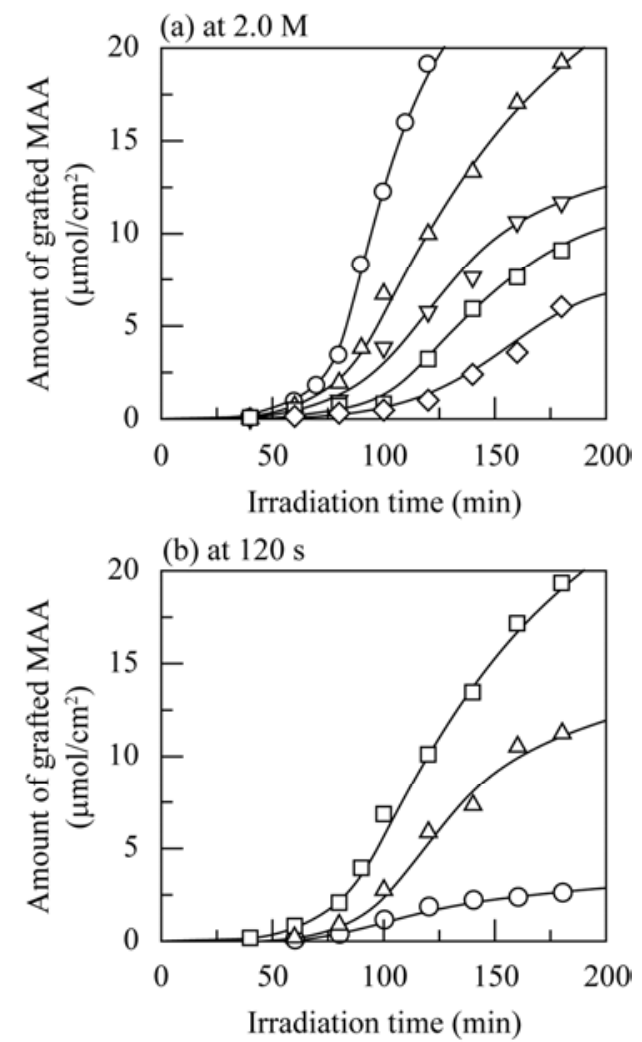

Figure 2. Changes in the grafted amount with the irradiation time for the photografting of MAA onto the PTFE plate at (a) $2.0 \mathrm{M}$ after the plasma treatment for $(\diamond)$ 10, ( $\square)$ 30, ( $\nabla) 60,(\Delta) 120$, and (०) $180 \mathrm{~s}$ and at (b) (O)

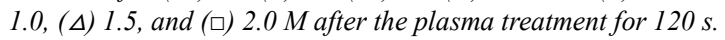

Figure 2 shows the changes in the grafted amount with the irradiation time for the PTFE-g-PMAA plates prepared (a) by the plasma treatment for $10-180 \mathrm{~s}$ and subsequent photografting at $2.0 \mathrm{M}$ and (b) by the plasma treatment for $120 \mathrm{~s}$ and subsequent photografting at 1.0-2.0 M. The grafted amount increased with the irradiation time. When the plasma treatment time and/or monomer concentration increased, the grafted amount further increased. It is extremely difficult to directly photograft MAA onto the PTFE surface without the plasma treatment mainly due to difficulty of the formation of active sites. Even if the PTFE surface was coated with BP without the plasma pretreatment, MAA was little photografted onto the PTFE plate (grafted amount $=0.15 \mu \mathrm{mol} / \mathrm{cm}^{2}$ ) $[38$, 49]. This result suggests that an oxygen-containing functional group generated by the plasma treatment acts as an active site for the photografting and the UV irradiation at $60^{\circ} \mathrm{C}$ during the photografting is an effective procedure to increase the grafted amount. Some of the studies on the combined use of the plasma treatment and grafting have reported that the grafting is initiated by thermal degradation of hydroperoxide groups, although there are arguments on the pros and cons of this notion [50-55].

\subsection{Water Absorptivity of Grafted Layers}

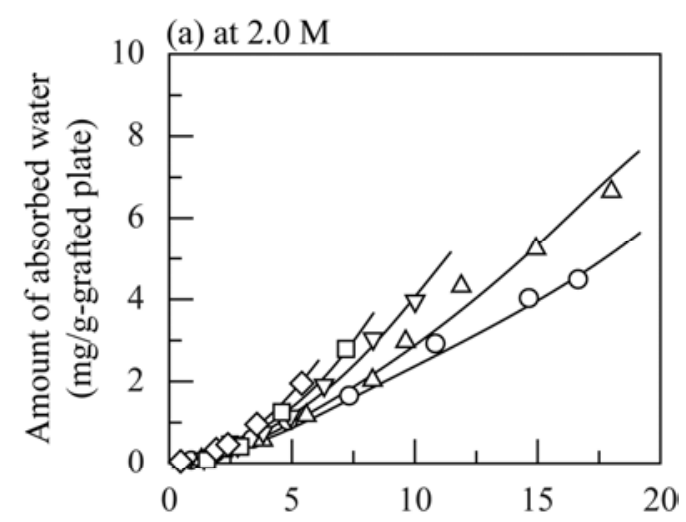

Amount of grafted MAA $\left(\mu \mathrm{mol} / \mathrm{cm}^{2}\right)$

(b) at $120 \mathrm{~s}$

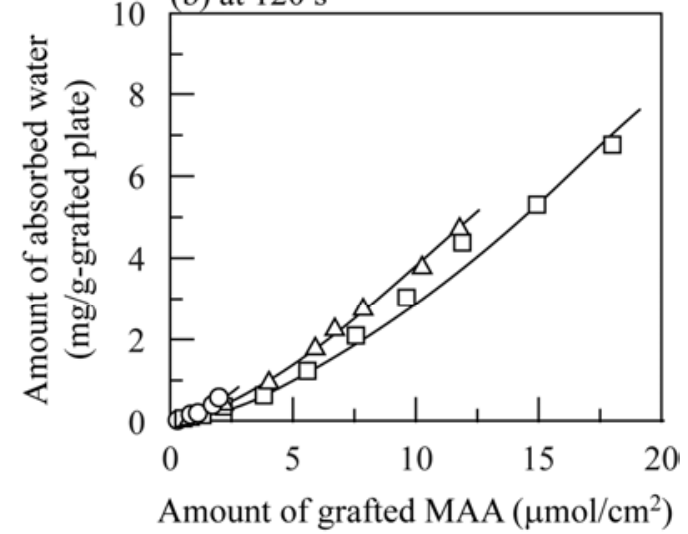

Figure 3. Changes in the amount of absorbed water with the grafted amount for the PTFE-g-PMAA plates prepared by the photografting at (a) $2.0 \mathrm{M}$ after the plasma treatment for $(\diamond)$ 10, ( $\square$ ) 30, ( $\nabla) 60,(\Delta) 120$, and (O) 180

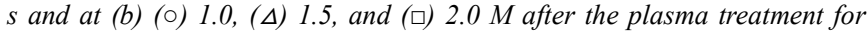
$120 \mathrm{~s}$. 
The water absorptivity of the grafted layers formed on the PTFE plates was measured. Figure 3 shows the amount of adsorbed water with the grafted amount for the PTFE-g-PMAA plates prepared by the photografting (a) at 2.0 $\mathrm{M}$ after the plasma treatment for 10-180 $\mathrm{s}$ and (b) at 1.0-2.0 M after the plasma treatment for $120 \mathrm{~s}$. The amount of absorbed water increased over the grafted amount, and as the plasma treatment time and/or the monomer concentration on the photografting decreased, the grafted layer possessed higher water absorptivity at lower grafted amounts. These results suggest that the water absorptivity of the grafted layer depends on the density of grafted PMAA chains in the grafted layer and

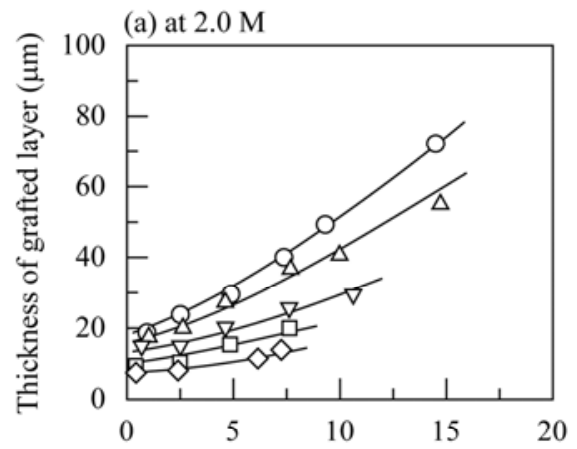

Amount of grafted MAA $\left(\mu \mathrm{mol} / \mathrm{cm}^{2}\right)$

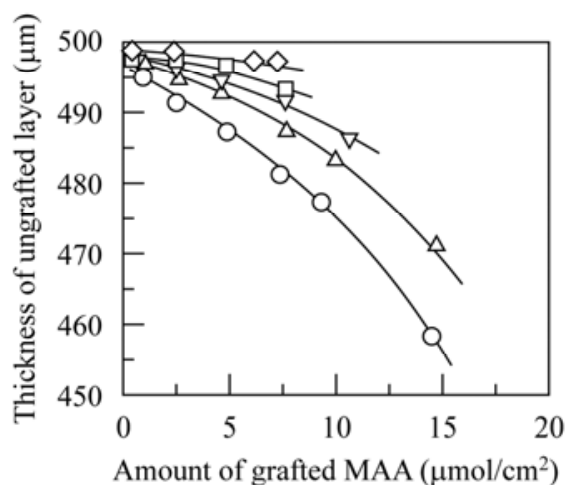

the location and thickness of the grafted layer as well as the length of grafted polymer chains $[21,22,26]$. Therefore, the thickness of the grafted layer was measured to discuss the effect of the grafting conditions on the water absorptivity of the grafted layer.

The grafted layers formed on the PTFE plates were stained with $\mathrm{TB}$, and then the thicknesses of the grafted and ungrafted layers were measured. Figure 4 shows the changes in the thicknesses of the grafted and ungrafted layers for the PTFE-g-PMAA plates prepared (a) at $2.0 \mathrm{M}$ after the plasma treatment for 10-180 $\mathrm{s}$ and (b) at 1.0-2.0 $\mathrm{M}$ after the plasma treatment for $120 \mathrm{~s}$.
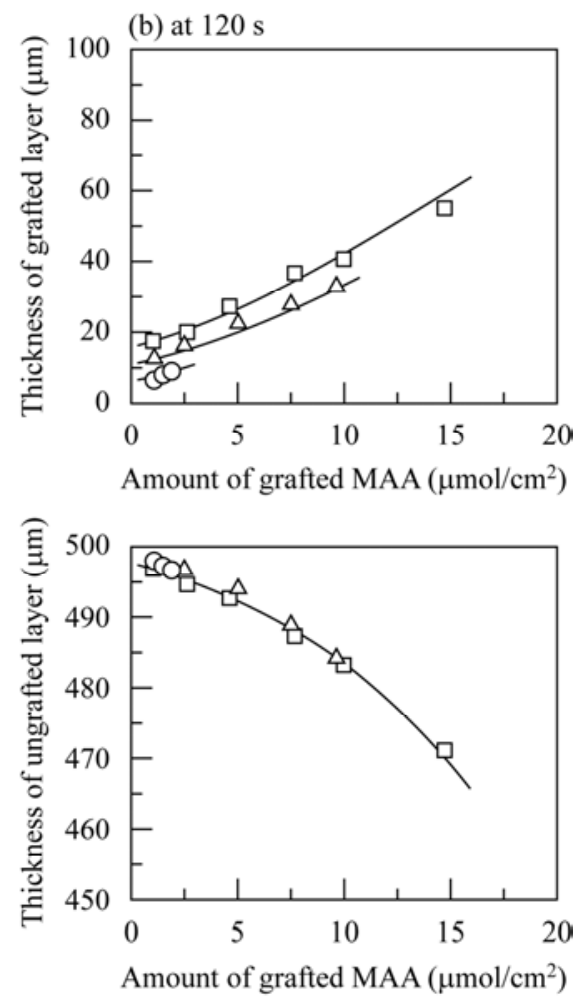

Figure 4. Changes in the thicknesses of the grafted layer and ungrafted layer with the grafted amount for the PTFE-g-PMAA plates prepared by the

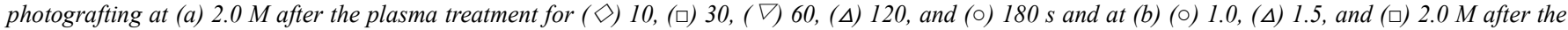
plasma treatment for $120 \mathrm{~s}$.

Figure 4 (a) shows that the thickness of the grafted layer increased over the grafted amount irrespective of the plasma treatment time. In addition, as the plasma treatment time was increased, thicker grafted layers were formed with the decreased thickness of the ungrafted layers. These results suggest that the location of photografting was restricted to the outer surface region and the grafted layer richer in grafted PMAA chains was formed. These factors are considered to give rise to higher water absorptivity of the grafted layers formed by the photografting after the plasma treatment for shorter times.

On the other hand, as the photografting was performed at higher monomer concentrations after the plasma treatment for $120 \mathrm{~s}$, the thicker grafted layers were formed on the PTFE surfaces. However, under these conditions, the decrease in the thickness of the ungrafted layers against the grafted amount was independent of the monomer concentrations on the photografting as shown in Figure 4 (b). In other words, it can be safely said that the grafted layers richer in grafted PMAA chains are formed at lower monomer concentrations. In addition, the length of grafted PMAA chains can be discussed based on the relation between the monomer concentration and the degree of polymerization according to the radical mechanism $[21,22]$, although it is impossible to determine the molecular weight of the grafted PMAA chains directly. Longer grafted polymer chains are considered to be formed at higher monomer concentrations. From the results in Figures 3 (b) and 4 (b), the grafted layers richer in grafted PMAA chains are considered to possess higher water absorptivity.

\subsection{Wettability of PTFE-g-PMAA Plates}

The surface modification of the PTFE plate by the photografting of MAA was also estimated from the water contact angle measurements. Figure 5 (a) shows the changes 
in the constant $\cos \theta$ value with the plasma treatment time before the photografting at $1.0-2.0 \mathrm{M}$. The $\cos \theta$ value increased with the grafted amount under the all grafting conditions. However, the constant $\cos \theta$ value was not obtained only for the PTFE-g-PMAA plates prepared by the plasma treatment for $10 \mathrm{~s}$ and subsequent photografting at 1.0 M. The grafted amounts at which the $\cos \theta$ values became constant and the constant $\cos \theta$ values were read out. Figure 5 (b) shows the changes in the grafted amount at which the $\cos \theta$ value became constant with the plasma treatment time before the photografting at $1.0-2.0 \mathrm{M}$. As the plasma treatment time was shortened, higher constant $\cos \theta$ value was obtained and the $\cos \theta$ value became constant at lower grafted amounts. In addition, as the monomer concentration on the photografting was lower, the $\cos \theta$ value became constant at lower grafted amounts. These results suggest that the location of photografting is restricted to the more outer surface region and more hydrophilic surfaces are formed, as the plasma treatment time was shorter and/or the monomer concentration was lower. In these cases, PTFE surfaces will be fully covered with grafted PMAA chains. On the other hand, for only the PTFE-g-PMAA plates prepared by the plasma treatment for $10 \mathrm{~s}$ and subsequent photografting at $1.0 \mathrm{M}$, the PTFE surface is yet to be covered with grafted PMAA chains.
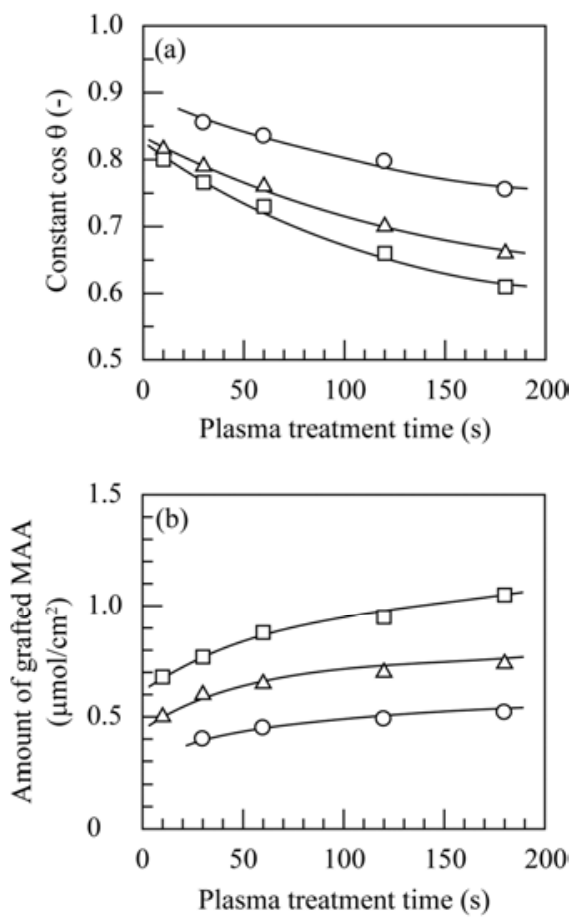

Figure 5. Changes in the (a) constant $\cos \theta$ value and (b) grafted amount at which the $\cos \theta$ value became constant with the plasma treatment time for

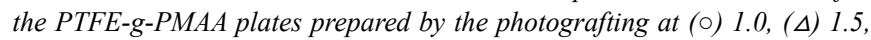
and () $2.0 \mathrm{M}$ after the plasma treatment for $120 \mathrm{~s}$.

\subsection{Adhesive Strength}

The adhesive strength of the PTFE-g-PMAA plates prepared under different grafting conditions above-mentioned was measured with a two-component type epoxy adhesive. Figure 6 shows the changes in the tensile shear adhesive strength with the grafted amount for the PTFE-g-PMAA plates prepared by the plasma treatment for different times and subsequent photografting at (a) 1.0, (b) 1.5, and (c) $2.0 \mathrm{M}$. When the PTFE plate was plasma-treated, the adhesive strength increased with the plasma treatment time from $52 \mathrm{kPa}$ for the untreated PTFE plate to $459 \mathrm{kPa}$ for the plasma treatment time for $180 \mathrm{~s}$ (data not shown). However, for these plasma-treated PTFE plates, no substrate breaking occurred. Meanwhile, the adhesive strength for the PTFE-g-PMAA plates increased with the grafted amount. In addition, the substrate breaking occurred, while the PTFE-g-PMAA plates were kept adhered to each other at higher grafted amounts (shaded symbols in Figure 6 (b) and (c)). This indicates that the adhesive strength exceeded the ultimate strength of the PTFE plate used.

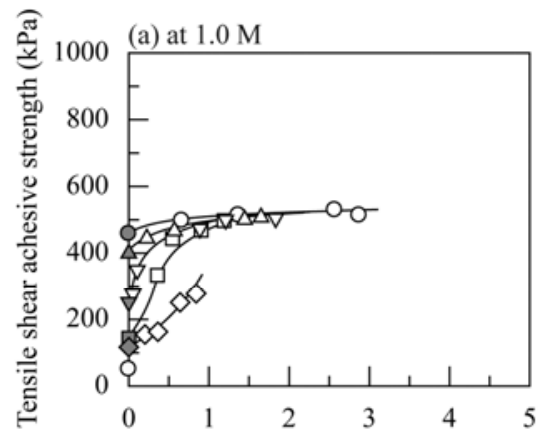

Amount of grafted MAA $\left(\mu \mathrm{mol} / \mathrm{cm}^{2}\right)$
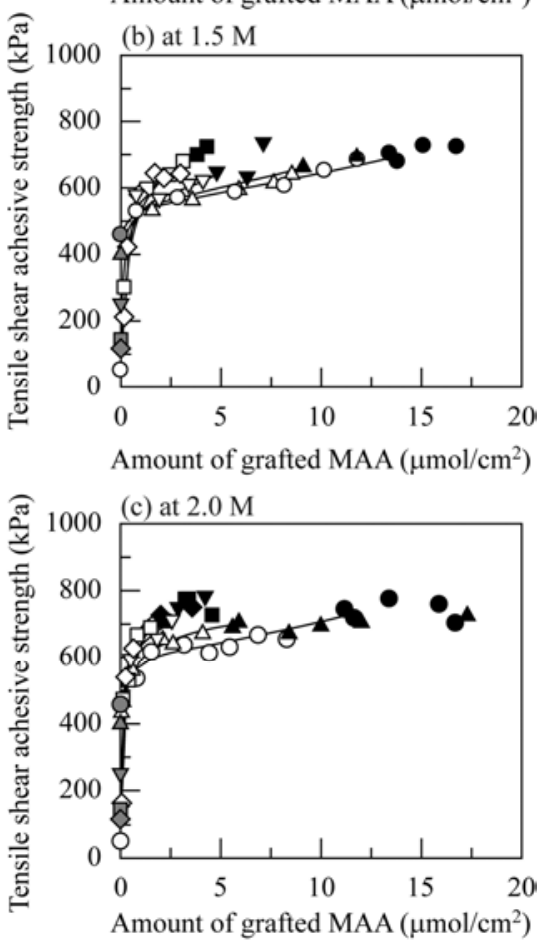

Figure 6. Changes in the tensile shear adhesive strength with the grafted amount for (open and shaded) ) the PTFE-g-PMAA plates prepared by the photografting at (a) 1.0, (b) 1.5, and (c) 2.0 Mafter (half-shaded) the plasma treatment for (०) 10, ( $\Delta)$ 30, ( $\nabla) 60$, ( $\square)$ 120, and ( $\diamond) 180 \mathrm{~s}$. The adhesive component was cured by applying the load of $0.5 \mathrm{~kg} / \mathrm{cm}^{2}$ at $60^{\circ} \mathrm{C}$, and open and shaded symbols represent cohesive strength and substrate breaking, respectively.

Figure 7 shows the changes in the minimum grafted amount 
at which the substrate breaking was observed with the plasma treatment time before the photografting. For the PTFE-g-PMAA plates prepared at 1.5 and $2.0 \mathrm{M}$, as the plasma treatment time before the photografting was shorter and/or the monomer concentration was higher, the PTFE plate was broken at lower grafted amounts. As shown in Figures 3 (a) and 5 (a), the grafted layers formed by the plasma treatment for shorter times and subsequent photografting possessed higher wettability and water-absorptivity. In addition, it was seen from Figure 5 (b) that as the plasma treatment time was shortened, the more hydrophilic grafted layer surfaces were formed at lower grafted amounts. These factors can facilitate the penetration of adhesives in the grafted layer, leading the substrate breaking at lower grafted amounts.

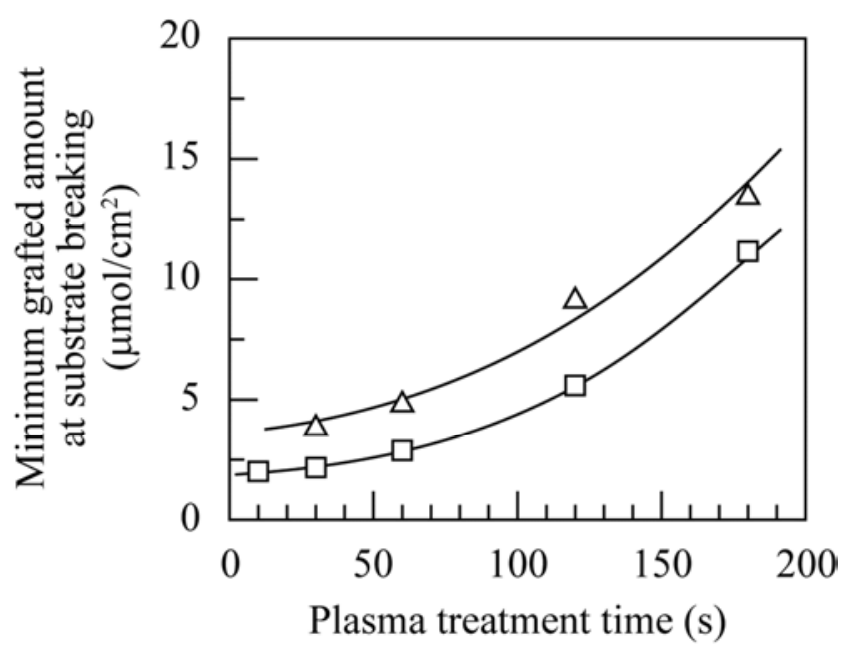

Figure 7. Changes in the grafted amount at which the substrate breaking occurred with the plasma treatment time for the PTFE-g-PMAA plates prepared by the photografting at $(\Delta) 1.5$ and (口) $2.0 \mathrm{M}$ after the plasma treatment.

On the other hand, it was also found from Figure 7 that the substrate breaking was not observed irrespective of the plasma treatment time for the photografting at $1.0 \mathrm{M}$ and only at the plasma treatment time of $10 \mathrm{~s}$ for the photografting at $1.5 \mathrm{M}$. Under these grafting conditions, the grafted amount limited to less than or comparable to $3 \mu \mathrm{mol} / \mathrm{cm}^{2}$. This suggests that the PTFE plate does not have a sufficient grafted amount for substrate breaking. In addition, the rate of monomer consumption for photografting, which was calculated from the slope of the grafted amount against the irradiation time in the steady state, increased from $0.028 \mathrm{mmol} / \mathrm{h}$ at $1.0 \mathrm{M}$ to 0.243 $\mathrm{mmol} / \mathrm{h}$ at $2.0 \mathrm{M}$ for the photografting on the PTFE plates plasma-treated for $120 \mathrm{~s}$ as seen in Figure 2. The photografting of MAA onto the plasma-treated PTFE plate in this study proceeds via the radical chain mechanism. Therefore, longer grafted polymer chains would form at higher monomer concentrations $[8,21]$. In addition, the discussion in relation with results from the image observation of the cross section shown in Figure 4 supports our suggestion that a high adhesive strength is obtained by penetration of adhesive components into a thick grafted layer formed by longer grafted polymer chains.

\subsection{Photografting at Different Temperatures}

The photografting of MAA onto the PTFE plates was performed at different temperatures after the plasma treatment for $120 \mathrm{~s}$ and adhesive properties of the PTFE-g-PMAA plates obtained were discussed in relation with their modified hydrophilic properties. The grafted amount increased over the irradiation time irrespective of the grafting temperature. As the grafting temperature was increased, higher grafted amounts were obtained at shorter irradiation times.

Figure 8 shows the changes in the amount of absorbed water with the grafted amount for the PTFE-g-PMAA plates prepared at $40-70^{\circ} \mathrm{C}$ (the initial MAA concentration was kept at $2.0 \mathrm{M}$ ) after the plasma treatment for $120 \mathrm{~s}$. The amount of absorbed water increased with the grafted amount, and the grafted layers with higher water absorptivity were formed at the PTFE surface, the photografting was performed at lower temperatures. Subsequently, contact angles for water were measured on these PTFE-g-PMAA plates.

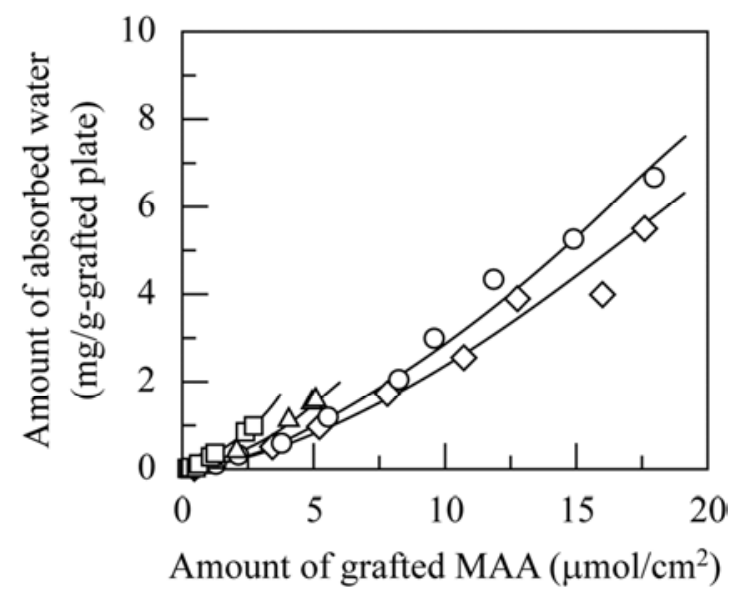

Figure 8. Changes in the amount of absorbed water with the grafted amount for the PTFE-g-PMAA plates prepared by the photografting at ( $\square$ ) 40, ( $\Delta$ ) 50 , (०) 60 , and $(\diamond) 70^{\circ} \mathrm{C}$ after the plasma treatment for $120 \mathrm{~s}$.

Figure 9 shows the changes in the constant $\cos \theta$ value obtained and the grafted amount at which the $\cos \theta$ value became constant with the grafting temperature. As the photografting was performed at lower temperatures, the $\cos \theta$ value became constant at lower grafted amounts and higher constant $\cos \theta$ values were obtained. The thickness of the grafted layer increased with the grafted amount and the thicker grafted layer was formed as shown in Figure 10. On the other hand, the thickness of the ungrafted layer increased with the grafted amount independent of the grafting temperature. This result means that the location of the photografting was independent of the grafting temperature. Meanwhile, as the grafting temperature decreased, longer grafted polymer chains will be formed mainly due to less chain transfer reaction under the conditions that the initial monomer concentration is kept constant. In addition, the adhesive strength for the PTFE-g-PMAA plates prepared at different grafting temperatures was measured. The adhesive strength increased and the substrate breaking occurred at all grafting temperatures. 


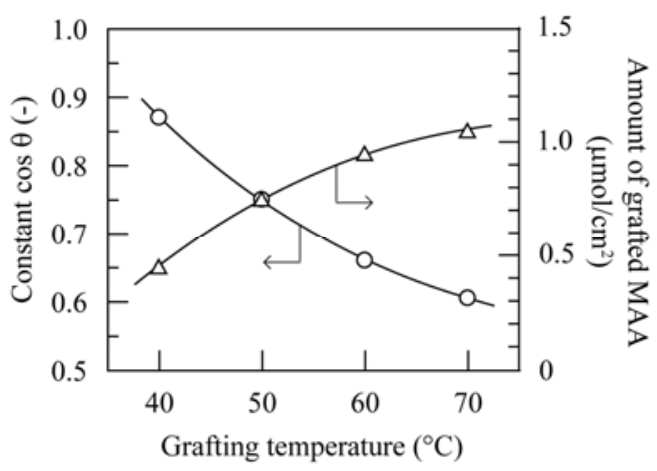

Figure 9. Changes in the (O) constant $\cos \theta$ value and ( $\Delta$ ) grafted amount at which the $\cos \theta$ value became constant with the grafting temperature for the PTFE-g-PMAA plates prepared by the photografting at $2.0 \mathrm{M}$ after the plasma treatment for $120 \mathrm{~s}$.

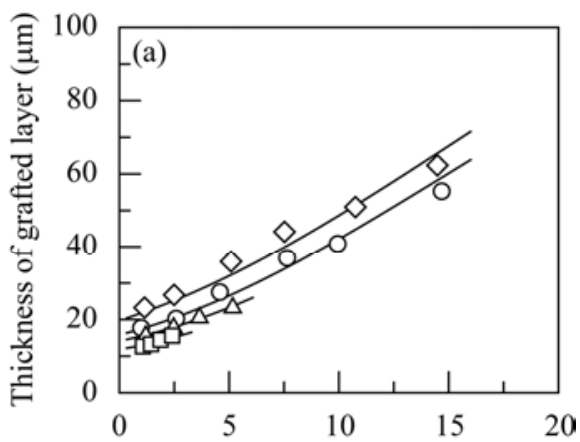

Amount of grafted MAA $\left(\mu \mathrm{mol} / \mathrm{cm}^{2}\right)$

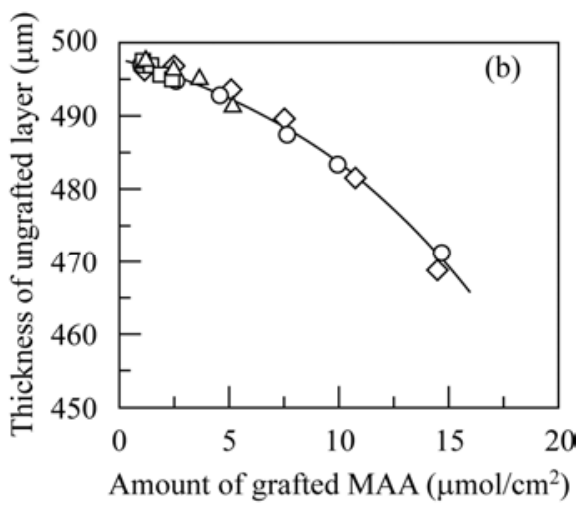

Figure 10. Changes in the thickness of the (a) grafted layer and (b) ungrafted layer with the grafted amount for the PTFE-g-PMAA plates prepared by the photografting at (ㅁ) $40,(\Delta) 50$, (०) 60 , and $(\diamond) 70^{\circ} \mathrm{C}$ after the plasma treatment for $120 \mathrm{~s}$.

Figure 11 shows the changes in the minimum grafted amount at which the substrate breaking occurred with the grafting temperature for the PTFE-g-PMAA plates prepared at 2.0 $\mathrm{M}$ after the plasma treatment for $120 \mathrm{~s}$. The substrate breaking occurred at lower grafted amounts for the PTFE-g-PMAA plates prepared at higher grafting temperatures. A following consideration was given on the basis of the above-described results. As the grafting temperature decreased, longer grafted polymer chains was formed and the grafted layers possessed higher water absorptivity. These factors would mutually contribute to an increase in the adhesive strength, leading to the decrease in the grafted amount at which the substrate breaking occurred with the decrease in the grafting temperature.

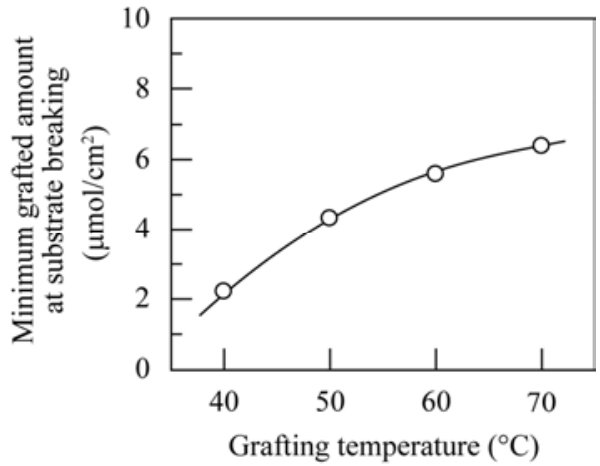

Figure 11. Change in the grafted amount at which the substrate breaking occurred with the grafting temperature for the PTFE-g-PMAA plates prepared by the photografting at $2.0 \mathrm{M}$ after the plasma treatment for $120 \mathrm{~s}$.

\section{Conclusion}

In this study, MAA was grafted onto the PTFE plate by the combined use of the plasma treatment and photografting. The effects of the grafting conditions, such as the plasma treatment time before the photografting and the monomer concentration and temperature on the photografting on the adhesive strength of the resultant PTFE-g-PMAA plates was discussed in relation to the wettability, water absorptivity, and location of grafting.

As the photografting of MAA was performed at lower monomer concentrations or at lower temperatures after the plasma treatment for shorter treatment times, higher wettability was obtained at lower grafted amounts and the grafted layer formed on the PTFE surface possessed higher water absorptivity. The adhesive strength increased with the grafted amount, and the substrate breaking was observed at lower grafted amounts as the plasma treatment time before the photografting was shorter for the photografting at 1.5 and $2.0 \mathrm{M}$. On the other hand, the substrate breaking was not observed for the photografting at $1.0 \mathrm{M}$ irrespective of the plasma treatment time and at $1.5 \mathrm{M}$ after the plasma treatment time of $10 \mathrm{~s}$. In addition, as the temperature on the photografting was lower, the PTFE-g-PMAA plate was broken at lower grafted amounts. The occurrence of the substrate breaking suggests that the adhesive strength exceeds the ultimate strength of the PTFE plate used. The substrate breaking was observed at the minimum grafted amount for the PTFE-g-PMAA plates prepared at $2.0 \mathrm{M}$ and $60^{\circ} \mathrm{C}$ after the plasma treatment for $10 \mathrm{~s}$ and at $2.0 \mathrm{M}$ and $40^{\circ} \mathrm{C}$ after the plasma treatment for $120 \mathrm{~s}$. The results obtained in this study support that the combination of the oxygen plasma treatment with photografting of MAA is an effective procedure to enhance the adhesivity of the PTFE surface.

\section{References}

[1] N. Inagaki, S. Tasaka, and Y. Goto, "Surface modification of poly(tetrafluoroethylene) film by plasma graft polymerization of sodium vinylsulfonate," J. Appl. Polym. Sci., Vol. 66, no. 6, pp. 77-84, 1997. 
[2] C. Y. Tu, Y. L. Liu, M. T. Luo, K. R. Lee, and J. Y. Lai, ChemPhysChem, "Effects of polymer architecture and composition on the adhesion of poly(tetrafluoroethylene)," Vol. 7, No. pp. 1355-1360, 2006.

[3] U. Lappan, H. M. Buchhammer, and K. Lunkwitz, "Surface modification of poly(tetrafluoroethylene) by plasma pretreatment and adsorption of polyelectrolytes," Polymer, Vol. 40, No. 14, pp. 4087-4091, 1999.

[4] Y. Chen, E. T. Kang, K. G. Neoh, and K. L. Tan, "Surface functionalization of poly(tetrafluoroethylene) films via consecutive graft copolymerization with glycidyl methacrylate and aniline," J. Phys. Chem. B, Vol. 104, No. 39, pp. 9171-9178, 2000 .

[5] E. M. Liston, L. Martinu, and M. R. Wertheimer, "Plasma surface modification of polymers for improved adhesion-a critical review," J. Adhes. Sci. Technol., Vol. 7, No. 10, pp. 1091-1127, 1993.

[6] N. Encinas, M. Pantoja, M. Torres-Remiro, and M. A. Martinez, "Approaches to Poly(Tetrafluoroethylene) Adhesive Bonding," J. Adhes., Vol. 87, No. 7-8, pp. 709-719, 2011.

[7] A. Vesel, M. Mozetic, and A. Zalar, "XPS characterization of PTFE after treatment with RF oxygen and nitrogen plasma," Surf. Interface Anal., Vol. 40, No. 3-4, pp. 661-663, 2008.

[8] Z. Kolská, A. Řezníčková, V. Hnatowicz, and V. Švorčík, "PTFE surface modification by $\mathrm{Ar}$ plasma and its characterization," Vacuum, Vol. 86, No. 7, pp. 643-647, 2012.

[9] G. Ren, Z. Zhang, X. Zhu, X. Men, W. Jiang, and W. Liu, "Combined effect of air-plasma treatment and lubricant filling on the dry sliding wear behavior of hybrid PTFE/Nomex fabric/phenolic composite," Compos. Sci. Technol., Vol. 100, No. 21, pp. 204-211, 2014.

[10] Y. Chen, Z. Zhao, J. Dai, and Y. Liu, "Topological and chemical investigation on super-hydrophobicity of PTFE surface caused by ion irradiation," Appl. Surf. Sci., Vol. 254, No. 2, pp. 464-467, 2007.

[11] J. Tian and Q. Xue, "Surface modification of PTFE by 60Co gamma-ray irradiation," J. Appl. Polym. Sci., Vol. 69, No. 3, pp. 435-441, 1998.

[12] N. M. Hidzir, D. J. T. Hill, D. Martin, and L. Grøndahl, "Radiation-induced grafting of acrylic acid onto expanded poly(tetrafluoroethylene) membranes," Polymer, Vol. 53, No. 26, pp. 6063-6071, 2012.

[13] E. Bucio, and G. Burillo, "Radiation grafting of $\mathrm{pH}$ and thermosensitive $\mathrm{N}$-isopropylacrylamide and acrylic acid onto PTFE films by two-steps process," Raidat. Phys. Chem., Vol. 76, No. 11-12, pp. 1724-1727, 2007.

[14] F. Rombaldoni, K. Mahmood, A. Varesano, M. B. Songia, A. Aluigi, C. Vineis, and G. Mazzuchetti, "Adhesion enhancement of electrospun nanofiber mats to polypropylene nonwoven fabric by low-temperature oxygen plasma treatment," Surf. Coat. Technol., Vol. 216, No. 13, pp. 178-184, 2013.

[15] S. Zanini, R. Barni, R. D. Pergola, and C. Riccardi, "Modification of the PTFE wettability by oxygen plasma treatments: influence of the operating parameters and investigation of the ageing behaviour," J. Phys. D. Appl. Phys., Vol. 47, No. 32, 325202 (9pp), 2014.

[16] H. Hunke, N. Soin, T. H. Shah, E. Kramer, A. Pascual, M. S. L.
Karuna, and E. Siores, "Low-Pressure $\mathrm{H}_{2}, \mathrm{NH}_{3}$ Microwave Plasma Treatment of Polytetrafluoroethylene (PTFE) Powders: Chemical, Thermal and Wettability Analysis," Materials, Vol. 8, No. 5, pp. 2258-2275, 2015.

[17] C. Corbella, S. Große-Kreul, and A. von Keudell, "Exploring the Structure of the Modified Top Layer of Polypropylene During Plasma Treatment," Plasma Process. Polym., Vol. 12, No. 6, pp. 564-573, 2015.

[18] M. Morra, E. Occhiello, and F. Garbassi, "Surface characterization of plasma-treated PTFE, Surf. Interface Sci., Vol. 16, No. 1-12, pp. 412-417, 1990.

[19] M. Morra, E. Occhiello, R. Marola, F. Garbasi, P. Humphrey, and D. J. Johnson, "On the aging of oxygen plasma-treated polydimethylsiloxane surfaces," J. Colloid Interface Sci., Vol. 137, No. 1, pp. 11-24, 1990.

[20] K. Yamada, S. Takeda, and M. Hirata, "Improvement of autohesive and adhesive properties of polyethylene plates by photografting with glycidyl methacrylate," J. Appl. Polym. Sci., Vol. 103, No. 1, pp. 493-500, 2007.

[21] K. Yamada, S. Yamagami, and Y. Naohara, "Estimation of surface properties of grafted layers formed on low- and high-density polyethylene plates by photografting of methacrylic acid and acrylic acid at different monomer concentrations and temperatures," J. Appl. Polym. Sci., Vol. 125, No. 4, pp. 2614-2625, 2012.

[22] H. Yoshida, K. Mochizuki, and K. Yamada, "Adhesion of ultrahigh molecular weight polyethylene plates photografted with hydrophilic monomers and evaluation of failure location by x-ray photoelectron spectroscopy," J. Appl. Polym. Sci., Vol. 131, No. 7, DOI:10 1002/app. 40133, 2014.

[23] K. Yamada, J. Kimura, and M. Hirata, "Autohesive properties of polyolefins photografted with hydrophilic monomers," $J$. Appl. Polym. Sci., Vol. 87, No. 14, 2244-2252 (2003).

[24] K. Yamada and M. Hiarta, "Autohesion of polyethylene plates by the photoinduced grafting of methaerylamide," ACS Symp. Ser., "Photoinitiated Polymerization", Vol. 847, pp. 511-521, 2003.

[25] K. Yamada, T. Kimura, H. Tsutaya, and M. Hirata, "Hydrophilic and adhesive properties of methacrylic acid-grafted polyethylene plates," J. Appl. Polym. Sci., Vol. 44, No. 6, pp. 993-1001, 1992.

[26] S. Yamagami, W. Kanama, and K. Yamada, "Adhesion of Polyethylene Plates Photografted with Methacrylic Acid and Acrylic Acid with Enzymatically Modified Chitosan Solutions and X-ray Photoelectron Spectroscopy Analysis of Failed Surfaces," J. Appl. Polym. Sci., Vol. 121, No. 2, pp. 939-950, 2011.

[27] K. Yamada, and Y. Naohara, "Adhesion of grafted polypropylene plates with enzymatically modified chitosan solutions and analysis of failed surfaces by X-ray photoelectron spectroscopy," J. Appl. Polym. Sci., Vol. 130, No. 2, pp. 1369-1376, 2013.

[28] M. M. Nasef, E. Shamsaei, H. Saidi, A. Ahmad, and K. Z. M. Dahlan, "Preparation and characterization of phosphoric acid composite membrane by radiation induced grafting of 4-vinylpyridine onto poly(ethylene-co-tetrafluoroethylene) followed by phosphoric acid foping," J. Appl. Polym. Sci., Vol. 128, No. 1, pp. 549-557, 2013. 
[29] N. M. El-Sawy and Z. I. Ali, "Iron(III) complexed with radiation-grafted acrylic acid onto poly (tetrafluoroethylene-co- perfluorovinyl ether) films," J. Appl. Polym. Sci., Vol. 103, No. 6, pp. 4065-4071, 2007.

[30] N. M. El-Sawy and F. A. Al Sacheer, "Physicochemical investigation of radiation-grafted poly(acrylic acid)-graftpoly(tetrafluoroethylene-ethylene) copolymer membranes and their use in metal recovery from aqueous solution," J. Appl. Polym. Sci., Vol. 85, No. 13, pp. 2692-2698, 2002.

[31] A. M. Dessouki, N. H. Taher, and M. B. El-Arnaouty, "Synthesis of permselective membranes by radiation-induced grafting of N-vinylpyrrolidone onto poly(tetrafluoroethylene hexafluoropropylene vinylidene fluoride) (TFB) films," Polym. Int., Vol. 48, No. 2, pp. 92-98, 1999.

[32] K. M. Baumgärtner, J. Schneider, A. Schulz, J. Feichtinger, and M. Walker, "Short-time plasma pre-treatment of polytetrafluoroethylene for improved adhesion," Surf. Coat. Technol., Vol. 142-144, pp. 501-506, 2001.

[33] T. Shi, M. Shao, H. Zhang, Q. Yang, and X. Shen, "Surface modification of porous poly(tetrafluoroethylene) film via cold plasma treatment," Appl. Surf. Sci., Vol. 258, No. 4, pp. 1474-1479, 2011.

[34] H. Xu, Z. Hu, S. Wu, and Y. Chen, "Surface modification of polytetrafluoroethylene by microwave plasma treatment of $\mathrm{H}_{2} \mathrm{O} /$ Ar mixture at low pressure," Mater. Chem. Phys., Vol. 80, No. 1, pp. 278-282, 2003.

[35] C. Wang and J. R. Chen, "Studies on surface graft polymerization of acrylic acid onto PTFE film by remote argon plasma initiation," Appl. Surf. Sci., Vol. 253, No. 10, pp. 4599-4606, 2007.

[36] S. Turmanova, M. Minchev, K. Vassilev, and G. Danev, "Surface grafting polymerization of vinyl monomers on poly(tetrafluoroethylene) films by plasma treatment," J. Polym. Res., Vol. 15, No. 4, pp. 309-318, 2008.

[37] K. Yamada, T. Ebihara, T. Gondo, K. Sakesegawa, and M. Hirata, "Membrane properties of porous and expanded poly(tetrafluoroethylene) films grafted with hydrophilic monomers and their permeation behavior," J. Appl. Polym. Sci., Vol. 61, No. 11, pp. 1899-1912, 1996.

[38] K. Yamada, J. Kimura, and M. Hirata, "Surface modification and autohesive properties of poly(tetrafluoroethylene) and polyethylene by the photografting technique," J. Photopolym. Sci. Technol., Vol. 11, No. 2, pp. 263-270, 1998.

[39] C. K. Na, H. J. Park, and B. J. Kim, "Optimal amidoximation conditions of acrylonitrile grafted onto polypropylene by photoirradiation-induced graft polymerization," J. Appl. Polym. Sci., Vol. 125, No. 1, pp. 776-785, 2012.

[40] S. Rattan and T. Sehgal, "Stimuli-responsive membranes through peroxidation radiation- induced grafting of 2-hydroxyethyl methacrylate (2-HEMA) onto isotactic polypropylene film (IPP)," J. Radioanal. Nucl. Chem., Vol. 293, No. 1, pp. 107-118, 2012.

[41] A. M. A. Ghaffar, M. B. El-Armaouty, M. E. Aboulfotouh, N. H. Taher, and A. A. Taha, "Radiation graft copolymerization of butyl methacrylate and acrylamide onto low density polyethylene and polypropylene films, and its application in wastewater treatment," Radiat. Eff. Def. Solids, Vol. 169, No. 9, pp. 741-753, 2014.
[42] T. A. Sherazi, T. Rehman, T, S. A. R. Naqvi, A. J. Shaikh, S. A. Shahzad, G. Abbas, R. Raza, and A. Waseem, "Surface functionalization of solid state ultra-high molecular weight polyethylene through chemical grafting," Appl. Surf. Sci., Vol. 359, No. 30, pp. 593-601, 2015.

[43] K. Yamada, Y. Saitoh, Y. Haga, K. Matsuda, and M. Hirata, "Adsorption and desorption properties of grafted polyethylene films modified with polyethylenimine chains," J. Appl. Polym. Sci., Vol. 102, No. 6, pp. 5965-5976, 2006.

[44] S. S. Mikhailova, O. M. Mykhaylyk, A. M. Dorfman, and V. I. Povstugar, "XPS study of finely dispersed iron powders modified by radiation-grafted acrylamide," Sur. Interface Anal., Vol. 29, No. 8, pp. 519-523, 2000 .

[45] A. Khot, A. Bailey, T. Debies, and G. A. Takacs, "XPS studies of poly(acrylic acid) grafted onto UV photo-oxidized polystyrene surfaces," J. Adhes. Sci. Technol., Vol. 26, No. 23, pp. 2627-2639, 2012.

[46] C. Y. Tu, Y. C. Wang, C. L. Li, K. R. Lee, J. Huang, and J. Y. Lai, "Expanded poly(tetrafluoroethylene) membrane surface modification using acetylene/nitrogen plasma treatment," Eur. Polym. J., Vol. 41, No. 10, pp. 2343-2353, 2005.

[47] S. Turmanova, M. Minchev, K. Vassilev, and G. Danev, "Surface grafting polymerization of vinyl monomers on poly(tetrafluoroethylene) films by plasma treatment," J. Polym. Res., Vol. 15, No. 4, pp. 309-318, 2008.

[48] N. M. Hidzir, D. J. T. Hill, E. Taran, D. Martin, L. Grøndahl, "Radiation-induced grafting of acrylic acid onto expanded poly(tetrafluoroethylene) membranes," Polymer, Vol. 53, No. 26, 6063-6071 (2012).

[49] M. Tachi, Y. Kimura, and K. Yamada, "Photografting of Methacrylic Acid onto Plasma-pretreated Poly (tetrafluoroethylene) Plates and Enhancement of Their Adhesivity," $J$. Photopolym. Sci. Technol., Vol. 28, No. 3, pp. 449-454, 2015.

[50] S. Saxena, A. R. Ray, B. Gupta, "Graft polymerization of Acrylic Acid onto Polypropylene Monofilament by RF Plasma," J. Appl. Polym. Sci., Vol. 116, no. 5, pp. 2884-2892, 2010.

[51] B. Gupta, K. Krishnanand, and B. L. Deopura, "Oxygen plasma-induced graft polymerization of acrylic acid on polycaprolactone monofilament," Eur. Polym. J., Vol. 48, No. 11, pp. 1940-1948, 2012.

[52] N. M. Hidzir, D. J. T. Hill, E. Taran, D. Martin, and L. Grøndahl, "Argon plasma treatment-induced grafting of acrylic acid onto expanded poly(tetrafluoroethylene) membranes," Polymer, Vol. 54, No. 24, pp. 6536-6546, 2013.

[53] X. Chi, H. Ohashi, and T. Yamaguchi, "Plasma-Induced graft polymerization inside pores of porous substrates assisted by an infiltration agent in acidic conditions," Plasma Process. Polym., Vol. 11, No. 4, pp. 306-314, 2014.

[54] S. Shi, Y. Zhou, X. Lu, Y. Ye, J. Huang, and X. Wang, "Plasma-Initiated DT Graft Polymerization of Acrylic Acid on Surface of Porous Polypropylene Membrane for Pore Size Control", Plasma Chem. Plasma Process, Vol. 34, No. 6, 1257-1269, 2014.

[55] F. Khelifa, S. Ershow, Y. Habibi, R. Snyders, and P. Dubois, "Free-Radical-Induced Grafting from Plasma Polymer Surfaces," Chem. Rev., Vol. 116, No. 6, pp. 3975-4005, 2016. 